\title{
Rehashing endocannabinoid antagonists: can we selectively target the periphery to safely treat obesity and type 2 diabetes?
}

\author{
Mary-Elizabeth Patti
}

Joslin Diabetes Center and Harvard Medical School, Boston, Massachusetts, USA.

\begin{abstract}
A growing body of evidence supports an important role for the endocannabinoid system as a regulator of appetite, body weight, and systemic metabolism, which is overactive in obesity and type 2 diabetes. While initial attempts to target this system using the cannabinoid receptor inverse agonist rimonabant were successful in producing modest weight loss and improving obesity-related metabolic complications in humans, adverse central nervous system side effects precluded introduction of this drug into clinical practice. However, new data, presented by Tam and colleagues in this issue of the JCI, demonstrate that selective blockade of peripheral cannabinoid receptors may be a novel successful therapeutic approach.
\end{abstract}

The active component in marijuana that is responsible for the increased hunger, increased food intake, and altered behavior experienced by those who use the drug is $\Delta^{9}$-tetrahydrocannabinol (THC). This discovery, in 1964 (1), triggered the quest for the endogenous THC receptor and signaling pathways that mediate these potent effects. Subsequently, two cannabinoid receptors, cannabinoid receptor 1 (CB1) and CB2, were isolated and cloned $(2,3)$. Both are $\mathrm{G}$ protein-coupled receptors (GPCRs), with widespread expression, including in the CNS, liver, gastrointestinal tract, pancreas, white and brown adipose tissue, skeletal muscle, and lymphocytes. CB1 is expressed in the CNS, liver, gastrointestinal tract, pancreas, white and brown adipose tissue, and skeletal muscle, while CB2 is expressed by lymphocytes, in the brain, and in skeletal muscle (Figure 1). Cannabinoids can also bind to the capsaicin/vanilloid receptor transient receptor potential cation channel, subfamily V, member 1 (TRPV1) (4). More recently, GPCR 55 (GPR55) has been identified as a potential atypical mediator of cannabinoid responses, as binding by cannabinoids and related lipids leads to intracellular calcium release, via RhoA and actin-dependent pathways (5), an intracellular signaling cascade distinct from that triggered by cannabinoid binding to $\mathrm{CB} 1$ and $\mathrm{CB} 2$.

Conflict of interest: The author acknowledges research grant support from the Lilly Foundation.

Citation for this article: J Clin Invest. 2010; 120(8):2646-2648. doi:10.1172/JCI44099.
Following the identification of cannabinoid receptors, it was logical to assume that there must also be endogenous ligands (endocannabinoids). Indeed, such ligands can be formed from arachidonic acid and other phospholipids within cellular membranes and include the lipid derivatives anandamide ( $N$-arachidonoylethanolamine [AEA]) and 2-arachidonoylglycerol (2-AG). 2-AG binds to both CB1 and CB2, while anandamide acts at other targets as well. Levels of endocannabinoids are governed not only by biosynthetic enzymes, but also by regulated degradation by fatty acid amide hydrolase (FAAH) and, in the case of 2-AG, by monoacylglycerol lipase (MAGL).

\section{Role of endocannabinoids in the central regulation of appetite and body weight}

Although endocannabinoids can modulate many physiological functions, including pain perception, drug dependence, and reward (6), it is the potent ability of endocannabinoids to regulate feeding behavior that has fueled attempts to harness this system for the treatment of obesity and its related complications. Both endogenous and exogenous cannabinoids increase appetite primarily by engaging CB1 in presynaptic neurons in the hypothalamus, modulating neurotransmitter release. The net effect of these signals is orexigenic (i.e., appetite stimulating) - achieved via both reduction in anorexigenic signals (e.g., corticotrophin-releasing hormone [CRH] and cocaine- and amphetamine-related transcript [CART]) and permissive effects on orexigenic signals (e.g., melanin-concentrating hormone $[\mathrm{MCH}])$. In parallel, endocannabinoids act on dopaminergic pathways in the limbic system to reinforce the hedonic effects of food intake.

Other appetite-regulatory signals also crosstalk with and modulate endocannabinoid levels and action (7). For example, leptin reduces synthesis and increases degradation of anandamide and 2-AG in the rat hypothalamus, and both leptin deficiency and resistance in rodents are associated with higher levels of endocannabinoids. Conversely, the orexigenic hormone ghrelin upregulates levels of endocannabinoids, and blockade of CB1 reduces ghrelin levels. Consistent with these data, modulation of endocannabinoid levels can potently alter food intake in rodents. CB1 antagonists reduce food intake, while inhibition of FAAH-mediated endocannabinoid catabolism increases food intake in rats.

\section{The promise and pitfalls of CB1 blockade with rimonabant}

Given the potent effects of cannabinoids on food intake, it is not surprising that pharmacologic blockade of the endocannabinoid system has long been considered a promising approach to the management of obesity and its complications. Indeed, in human clinical trials, the CB1 inverse agonist rimonabant consistently increased weight loss by $4-6 \mathrm{~kg}$ over $6-12$ months, as compared with placebo, with accompanying improvements in the insulin resistance, hyperlipidemia, and diabetes metabolic control of the participants $(8,9)$. However, given that the dominant mechanism of action of rimonabant was to reduce appetite via CNS pathways, concerns remained regarding the potential for side effects, including depression and anxiety. While these adverse effects did not achieve the level of statistical significance in individual trials, subsequent meta-analyses demonstrated that rimonabant increased the 


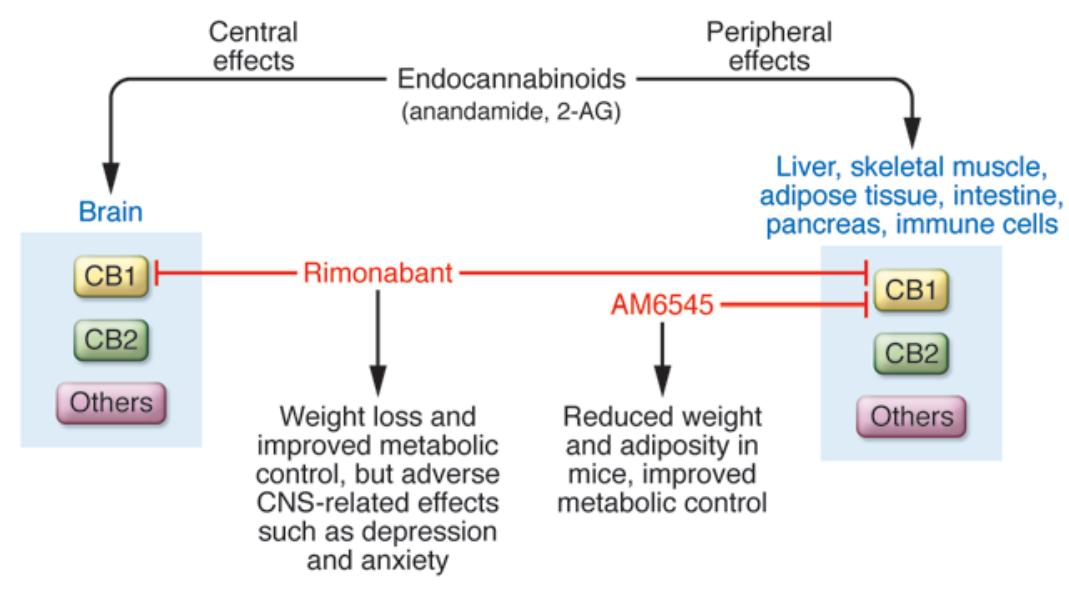

Figure 1

Targeting the endocannabinoid system. Endogenous cannabinoids bind to CB1 in both the CNS and multiple peripheral tissues. Rimonabant, a CB1 inverse agonist, inhibits cannabinoid signaling both centrally and peripherally. In clinical trials, it consistently increased weight loss, as compared with placebo, and improved metabolic control. However, due to concerns about adverse CNS-mediated effects such as depression and anxiety, rimonabant was withdrawn from the worldwide market. In contrast, AM6545 does not penetrate the CNS and inhibits cannabinoid signaling only in the periphery (19). It exerts beneficial metabolic effects in mice.

risk of serious adverse events, including the need to discontinue the drug due to anxiety or depression (9). Since clinical trials typically exclude patients with preexisting psychiatric disease, concerns remained about the safety of rimonabant if therapy were to be extended to a broader clinical population, and the drug was withdrawn from the worldwide market.

\section{Peripheral targets of endocannabinoid metabolism?}

Despite the ultimately disappointing adverse outcomes of treatment with rimonabant, emerging evidence supports the idea that cannabinoid receptors also play important roles in peripheral tissues and thus may be targeted by a selective approach $(6,7)$. Support for this perspective comes from several sources. First, cannabinoid receptor pathway components are expressed and active in peripheral tissues including the liver (10), adipose (11, 12), skeletal muscle (13), intestine (14), and pancreas (15), all of which are critical for the maintenance of normal glucose homeostasis. Second, cannabinoid activity is increased in both obese rodents and humans, potentially contributing to dysregulation of metabolism $(11,16)$. Last, centrally mediated reduction in appetite and food intake cannot fully explain the beneficial metabolic effects of cannabinoid receptor blockade. For example, mice treated with a CB1 antagonist show sus- tained decreases in body weight, despite only transient reductions in food intake (17); similarly, mice lacking CB1 are resistant to dietary obesity, despite caloric intake similar to that of wild-type animals (18). Thus, interrupting endocannabinoid action selectively in peripheral tissues has been suggested as an approach to treat the metabolic consequences of obesity and related diseases, including type 2 diabetes, without causing the undesirable CNS effects that occurred with rimonabant. The studies of Tam and colleagues, in this issue of the JCI provide key data to address this timely hypothesis (19).

\section{Evidence for efficacy of peripheral CB1 blockade}

Tam et al. first developed a CB1 antagonist (AM6545) that retained binding activity but did not penetrate the brain due to its reduced lipid solubility (19). In contrast to rimonabant, which was detectable in brain and blocked cannabinoid-mediated reductions in activity and hypothermia, AM6545 was ineffective at blocking these CNS-mediated behavioral effects of cannabinoid agonists. Remarkably, however, peripherally active AM6545 reduced body weight and adiposity in mice with diet-induced obesity. AM6545 also markedly improved systemic metabolism, as indicated by reduced fasting glucose, improved glucose tolerance, reduced insulin and leptin levels, increased adiponectin levels, and reduced hepatic tri- glyceride content in mice with diet-induced obesity. Most of these effects were qualitatively similar, but somewhat less robust, than those of rimonabant, perhaps reflecting greater potency of or additional centrally mediated effects of rimonabant on food intake or metabolism.

\section{Weight loss-independent effects of peripheral CB1 blockade}

Several important questions arise from these data. Are these effects independent of weight loss? While AM6545 did modestly reduce food intake early in the course of treatment, improvements in metabolic parameters were not observed in pair-fed controls, suggesting that the effects of AM6545 were largely mediated by increases in energy expenditure. Moreover, beneficial metabolic effects were also observed in leptin-deficient $o b / o b$ mice, despite no effects on body weight or fat, again indicating that weight loss is not required for the therapeutic effects of peripheral CB1 inhibition.

Which peripheral tissues are playing the dominant role in efficacy of CB1 blockade by AM6545? Hepatic effects are likely dominant, as evidenced by more striking reductions in fasting glucose levels, robust reductions in hepatic triglyceride content, reduced expression and function of lipogenic genes, and increased triglyceride secretion (19). These conclusions are in accordance with the authors' previous work demonstrating that liver-specific deletion of CB1 could protect mice from diet-induced glucose intolerance and hepatic steatosis (10). In the current study (19), liver-specific transgenic expression of hepatic CB1 was sufficient to induce glucose intolerance and confer sensitivity to AM6545, also pointing to prominent liver effects of endocannabinoid receptor signaling. It is likely that additional tissues, including adipose tissue, also play a role in mediating the therapeutic effects of CB1 inhibition, as indicated by reductions in lipogenic gene expression in subcutaneous and visceral fat in treated mice (19). To fully resolve the relative importance of liver, adipose, and other tissues in mediating cannabinoid signals and responses to pharmacotherapy, future studies on additional tissue-specific mutant mice are required.

\section{Conclusions and future directions}

The work of Tam and colleagues (19) provides support for the emerging concept that peripheral endocannabinoid signaling is altered in obesity and can contribute 
substantially to important metabolic complications, such as type 2 diabetes and fatty liver. Given the urgent need for more effective therapies for these common diseases, it will be essential to identify molecular mediators of increased endocannabinoid activity in obesity. As with many complex diseases, it is likely that endocannabinoid dysregulation in obesity will arise at the intersection between genetic susceptibility (e.g., polymorphisms in endocannabinoid pathway genes) and an obesogenic metabolic environment, including overnutrition, suboptimal dietary lipid composition, inactivity, insulin/leptin resistance, and tissue inflammation. Studies are required to both fully dissect these possibilities and to identify optimal dietary and lifestyle interventions useful to attenuate endocannabinoid overactivity (20). Parallel identification of additional pharmacologic compounds that can reduce activity of the cannabinoid system is clearly needed. However, the critical challenge will be to verify the absence of CNS effects of endocannabinoid modulation in longer-term studies in both preclinical models and humans, so that we are not doomed to rehash the unfortunate saga of rimonabant.

\section{Acknowledgments}

The author gratefully acknowledges research support from NIH grant DK062948 (to M.E. Patti), the American
Diabetes Association, the Lilly Foundation, and the Graetz Fund.

Address correspondence to: Mary-Elizabeth Patti, Joslin Diabetes Center, Boston, Massachusetts 02215, USA. Phone: 617.735.1966; Fax: 617.732.2593; E-mail: mary.elizabeth.patti@joslin.harvard.edu.

1. Gaoni Y, Mechoulam R. Isolation, structure, and partial synthesis of an active constituent of hashish. J Am Chem Soc. 1964;86(8):1646-1647.

2. Matsuda LA, Lolait SJ, Brownstein MJ, Young AC, Bonner TI. Structure of a cannabinoid receptor and functional expression of the cloned cDNA. Nature. 1990;346(6284):561-564.

3. Munro S, Thomas KL, Abu-Shaar M. Molecular characterization of a peripheral receptor for cannabinoids. Nature. 1993;365(6441):61-65.

4. Premkumar LS, Ahern GP. Induction of vanilloid receptor channel activity by protein kinase C. Nature. 2000;408(6815):985-990.

5. Lauckner JE, Jense JB, Chen H-C, Lu H-C, Hille B, Mackie K. GPR 55 is a cannabinoid receptor that increases intracellular calcium and inhibits $M$ current. Proc Natl Acad Sci U S A. 2008;105(7):2699-2704.

6. Di Marzo V. Targeting the endocannabinoid system: to enhance or reduce? Nat Rev Drug Discov. 2008;7(5):438-455.

7. Lambert DM, Muccioli GG. Endocannabinoids and related $\mathrm{N}$-acylethanolamines in the control of appetite and energy metabolism: emergence of new molecular players. Curr Opin Clin Nutr Metab Care. 2007;10(6):735-744.

8. Scheen AJ, Finer N, Hollander P, Jensen MD, Van Gaal LF. Efficacy and tolerability of rimonabant in overweight or obese patients with type 2 diabetes: a randomised controlled study. Lancet. 2006;368(9548):1660-1672.

9. Christensen R, Kristensen PK, Bartels EM, Bliddal $\mathrm{H}$, Astrup A. Efficacy and safety of the weight-loss drug rimonabant: a meta-analysis of randomised trials. Lancet. 2007;370(9600):1706-1713.

10. Osei-Hyiaman D, et al. Hepatic CB1 receptor is required for development of diet-induced steatosis, dyslipidemia, and insulin and leptin resistance in mice. J Clin Invest. 2008;118(9):3160-3169.

11. Bluher $M$, et al. Dysregulation of the peripheral and adipose tissue endocannabinoid system in human abdominal obesity. Diabetes. 2006;55(11):3053-3060.

12. Motaghedi R, McGraw TE. The CB1 endocannabinoid system modulates adipocyte insulin sensitivity. Obesity (Silver Spring). 2008;16(8):1727-1734.

13. Cavuoto P, McAinch AJ, Hatzinikolas G, CameronSmith D, Wittert GA. Effects of cannabinoid receptors on skeletal muscle oxidative pathways. Mol Cell Endocrinol. 2007;267(1-2):63-69.

14. Artmann A, et al. Influence of dietary fatty acids on endocannabinoid and $\mathrm{N}$-acylethanolamine levels in rat brain, liver and small intestine. Biochim Biophys Acta. 2008;1781(4):200-212.

15. Bermudez-Silva FJ, Suarez PJ, Nadal A, Rodriguez de Fonseca F. The role of the pancreatic endocannabinoid system in glucose metabolism. Best Pract Res Clin Endocrinol Metab. 2009;23(1):87-102.

16. Engeli S, et al. Activation of the peripheral endocannabinoid system in human obesity. Diabetes. 2005;54(10):2838-2843.

17. Ravinet TC, et al. Anti-obesity effect of SR141716, a CB1 receptor antagonist, in diet-induced obese mice. Am J Physiol Regul Integr Comp Physiol. 2003; 284(2):R345-R353

18. Ravinet TC, Delgorge C, Menet C, Arnone M, Soubrie P. CB1 cannabinoid receptor knockout in mice leads to leanness, resistance to diet-induced obesity and enhanced leptin sensitivity. Int J Obes Relat Metab Disord. 2004;28(4):640-648.

19. Tam J, et al. Peripheral CB1 cannabinoid receptor blockade improves cardiometabolic risk in mouse models of obesity. J Clin Invest. 2010; 120(8):2953-2966.

20. Di Marzo V, et al. Changes in plasma endocannabinoid levels in viscerally obese men following a 1 year lifestyle modification programme and waist circumference reduction: associations with changes in metabolic risk factors. Diabetologia. 2009;52(2):213-217.

\title{
Mice that "conditionally" lack basophils, AT LAST
}

\section{Booki Min}

\author{
Department of Immunology, Lerner Research Institute, Cleveland Clinic Foundation, Cleveland, Ohio, USA.
}

\begin{abstract}
Basophils are the least abundant granulocytes found in the circulation. Until recently, their functions were poorly understood. In the past few years, the list of basophil functions in the context of immunity has dramatically increased. Thus, the need for basophil-deficient animal models to confirm these findings is imperative. In this issue of the JCI, Wada and colleagues introduce the first mouse model in which basophils are conditionally ablated in vivo. Using this model, they then uncover a nonredundant role for basophils in acquired immunity against tick infection.
\end{abstract}

Basophils, the least abundant granulocytes found in the circulation, have long been out-

Conflict of interest: The author has declared that no conflict of interest exists.

Citation for this article: J Clin Invest. 2010; 120(8):2648-2651. doi:10.1172/JCI44058. siders in the immunology community. This is in part because of their scarcity and phenotypic similarity to mast cells. Although mast cells are not classified as granulocytes, they share several characteristics with basophils; these include surface expression of the high-affinity $\mathrm{Fc}$ receptor for $\operatorname{IgE}$ ( $\mathrm{Fc \varepsilon RI}$ ) and the ability to release chemical mediators such as histamine after stimulation. In mice, mature basophils have a relatively small cytoplasm and few cytoplasmic granules (1). However, surface markers that are specific for basophils have not been identified; thus, an experimental tool to examine the unique functions of basophils in vivo has long been wanted. Recent studies have increased the need for this, as they have accumulated convincing evidence that basophils play crucial roles in the development of immune responses, particularly those associated with type 2 immunity $(2,3)$.

Basophil-mediated regulation of immune responses is primarily medi- 